\title{
C-REACTIVE PROTEIN IN AUTOLOGOUS STEM CELL TRANSPLANTATION: PREDICTION OF CLINICAL COMPLICATION
}

\author{
Bruna Sabioni', Eduardo Edelman Saul', Rodrigo Doyle Portugal', Marcia Rejane da Silva Valetim², \\ Angelo Maiolino ${ }^{1,3}$, Marcia Garnica ${ }^{1,2^{*}}$

\begin{abstract}
1 Universidade Federal do Rio de Janeiro, Department of Internal Medicine, 2 Complexo Hospitalar de Niterói , 3 Américas Centro de Oncologia Integrado
\end{abstract}

Running title: C-REACTIVE IN AUTOLOGOUS TRANSPLANTATION

Correspondence to: Marcia Garnica (marciagarnica@hucff.ufrj.br)

\begin{abstract}
Objective: The aim of this study was to evaluate C-reactive protein (CRP) as a predictor of complications during autologous stem cell transplant (HSCT). Methods: We analyzed a cohort of 340 transplants. Correlation analyses were performed, including CRP obtained before HSCT, on Day+3, Day+6, Day+9, after Day+11, and at the onset of febrile neutropenia, and the following outcomes: bacteremia, severity of mucositis, length of neutropenia and hospitalization, and death. Results: the median age was 54 years old (ranging from 20 to 75), and $62 \%$ and $20 \%$ were multiple myeloma and non-Hodgkin lymphoma cases, respectively. The median CRP levels increased from D+3 to D+9 and after that decreased progressively until discharge. CRP levels were associated with bacteremia, mucositis grade, length of neutropenia and hospitalization, and death. Variation in CRP values from D+3 to D+6 predicted complications. Mortality was associated with $D+9$ CRP levels ( 19 vs. $7.9 \mathrm{mg} / \mathrm{dL} ; \mathrm{p}<0.01$ ), and a ROC curve area of $0.83(95 \% \mathrm{Cl} 0.7-0.95)$ to predict mortality. At a cut-off of $8.5 \mathrm{mg} / \mathrm{dL}, \mathrm{D}+9$ CRP had $83 \%$ and $79 \%$ sensitivity and specificity, respectively.

Conclusions: In this study, CRP dynamics were associated with several HSCT complications. CRP levels curve could be applied to indicate poor outcomes during HSCT.
\end{abstract}

Keywords: autologous stem cell transplant; complication; febrile neutropenia; C-reactive protein

\section{INTRODUCTION}

Hematopoietic stem cell transplant (HSCT) is an essential and potentially curative treatment option for several malignant hematological disorders. It is considered standard of care for multiple myeloma patients and rescue therapy in relapsed lymphoma patients. Although HSCT has been considered a safety procedure compared to other types of transplant, multicenter cohorts had reported the mortality related to this procedure to be around 2-5\% ${ }^{1}$. The most prevalent cause of death is related to infection episodes, mainly bacterial sepsis during neutropenia ${ }^{2,3}$. Mucositis is another frequent and sometimes severe complication of HSCT conditioning chemotherapy.
Although mucositis is not commonly directly related to mortality, the severity of mucosal damage is a significant risk factor for infection, bleeding and contributes for prolonged hospitalization length, higher costs and worst quality of life ${ }^{4,5}$.

Some serum biomarkers, such as procalcitonin, IL-6, and C-reactive protein (CRP) have been applied in order to early identify potential clinical complications and to guide medical staff to intensify clinical support for those in high-risk. Their impact in predicting outcomes was validated in critical care patients, and 
neutropenic patients ${ }^{6-8}$. Also, C-reactive protein is a widely used biomarker, and considered a low-cost exam.

In this study, we describe the dynamics of CRP during HSCT and its correlation with pre-transplant characteristics, and infectious and non-infectious clinical outcomes.

\section{METHODS}

This observational study was conducted in two centers (Hospital Universitário Clementino Fraga Filho [HUCFF], Federal University of Rio de Janeiro, Brazil, and Complexo Hospitalar de Niteroi [CHN]. HUCFF is a tertiary care hospital with 200 beds, including a hematology and hematopoietic cell transplant (HSCT) unit with eight single-bed rooms equipped with high-efficiency particulate air (HEPA) filter and positive pressure, and five double-bed rooms without HEPA filter. CHN is a tertiary care hospital with $\sim 400$ beds, including a hematology and hematopoietic cell transplant (HCT) unit with eight single-bed rooms equipped with HEPA filter and positive pressure, and 12 single-bed rooms without HEPA filter. Both institutions' Ethical Committees approved this study ("Comitê de Ética em Pesquisa do Hospital Universitário Clementino Fraga Filho" and "ProCEP Comitê de ética em Pesquisa da ESHO Empresa de Serviços Hospitalares - Hospital Pro-Cardiaco"). All procedures followed were in accordance with the ethical standards of the responsible committee on human experimentation (institutional and national) and with the Helsinki Declaration of 1975, as revised in 2008.

For this analysis, we selected all patients admitted between 2012 and 2016 who fulfilled the following criteria: a) high dose chemotherapy for HSCT conditioning; $b$ ) at least two measures of CRP performed during the HSCT hospitalization period; and c) at least 18 years old. Patients were included more than once when submitted to a second HSCT during the period of enrollment. Patients were followed from the conditioning period until discharge after engraftment or death. The cohort was described regarding demographic characteristics (age, gender), underlying disease and type of conditioning regimen. The following clinical outcomes were analyzed: 1) bacteremia, 2) severe mucositis (by the Common Toxicity Criteria of National Cancer Institute World Health Organization), 3) length of neutropenia 4) length of hospitalization, and 5) death. All data were collected prospectively, as part of an extensive database of stem cell transplant recipients.
Neutropenia was defined as an absolute neutrophil count (ANC) $<500 / \mathrm{mm} 3$, and Bone marrow recovery as at least two consecutive ANC $>500 / \mathrm{mm} 3$, obtained on two different days. Grade 3 or 4 were considered severe mucositis. Fever was defined as an axillary temperature $\geq 380 \mathrm{C}$. In the case of fever during neutropenia (febrile neutropenia), blood cultures were drawn, and the patients were immediately started on intravenous (IV) Cefepime unless a previous episode of febrile neutropenia documented a Cefepime-resistant Gram-negative organism. In this case, a carbapenem (imipenem or meropenem) was started. Blood cultures were repeated if the fever persisted, recurred, or as clinically indicated. Modifications in the empirical antibiotic regimen were performed according to cultures' results and the patient's clinical course. Additionally, febrile neutropenia episodes were defined as fever of unknown origin (FUO), bacteremia, microbiologically documented infection without bacteremia, or clinically documented infection. Bacteremia was defined as presence of positive blood culture either with a single organism or polymicrobial infection. Microbiologically documented infection without bacteremia was defined as the presence of pathogen in fluid or tissue suspected to be involved with the infection. Clinically documented infection was when a site of infection was diagnosed by signs or radiological features (e.g., cellulitis, pneumonia) but no microbiological documentation was achieved.

CRP values were expressed in $\mathrm{mg} / \mathrm{dL}$, and the negative reference value by the manufacturer is was < $0.3 \mathrm{mg} / \mathrm{dL}$. As an observational study, CRP samples were drawn at the discretion of clinicians. For the present study, we analyzed CRP values collected in the following interval periods, related to the stem cell reinfusion ("D Zero"):

$$
\begin{aligned}
& \text { - before HSCT CRP: admission day until D-2 } \\
& \text { - D Zero CRP: D-1 until D+1 } \\
& \text { - D+3 CRP: D+2 until D+4 } \\
& \text { - D+6 CRP: D+5 until D+7 } \\
& \text { - D+9 CRP: D+8 until D+10 } \\
& \text { - After D+11 CRP: after D+11 until discharge }
\end{aligned}
$$

Finally, samples were drawn close to febrile neutropenia (FN CRP) and engraftment (engraftment CRP) were also included in the analysis.

CRP single values and the dynamic of values variation between interval periods were analyzed as outcomes' predictors. All statistical analyses were performed using the SPSS for Windows software (version 21.0.1, SPSS, Inc., USA). The Chi-square test was used to compare proportions, and the Mann-Whitney test 
to compare continuous variables; Spearman test was used to correlation analyses, and Receiver Operating Characteristic (ROC) curve to define sensitivity and specificity values. $P$ values $<0.05$ were considered statistically significant.

\section{RESULTS}

A total of 340 stem cell transplants were performed during the study period in 338 patients. Two patients had two HSCT performed. The median age of the group was 54 years old (ranging from 20 to 75), and $53 \%$ were male. Baseline diseases were more frequently Multiple Myeloma (62\%), Non-Hodgkin Lymphoma (20\%), and Hodgkin Lymphoma $17 \%$. Three patients had other baseline diseases (Acute Myeloid Leukemia and germinative tumor in 1 and 2 cases, respectively). Demographic and clinical characteristics are shown in Table 1. Febrile neutropenia (FN) was documented in 299 (88\%), and bacteremia in 80 cases $(26,7 \%$ of FN). Severe mucositis was observed in $26 \%$ of patients. Seven patients from the cohort died during hospitalization (2\%).

A total of 1761 CRP tests were included in the analyzes. The median samples collection per patient was 5, ranging from 2 to 7 . At admission, 158 (66\%) patients had CRP levels above the normal reference. The median level of Before HSCT CRP was $0.48 \mathrm{mg} /$ $\mathrm{dL}$ (ranging from $<0.01$ to 15 ), and decreased on $\mathrm{D}$ Zero (median $0.38 \mathrm{md} / \mathrm{dL})(\mathrm{p}<0.01)$. (Table 2$)$ In the post-transplant period, the median variation from $\mathrm{D}+3$ to $\mathrm{D}+6 \mathrm{CRP}$ was $3.23 \mathrm{mg} / \mathrm{dL}(-7.6-+31)$ which represented a fourfold increase, and only $11 \%$ of patients had a decrease in CRP values on this period. After $D+9$ and engraftment, there was a decrease in the CRP median $(p<0.01)$. On discharge, CRP medians remained higher than on D Zero levels $(p<0.01)$. (Figure 1)

Febrile neutropenia was documented in 299 transplant recipients. Only in one patient, FN CRP was not collected at the onset of FN.CRP levels at the onset of $\mathrm{FN}$ were higher in patients with documented bacteremia compared to others ( $5.5 \mathrm{vs} .2 .69 \mathrm{mg} / \mathrm{dL}$, respectively: $p=0.01$ ). When testing FN CRP to predict bacteremia using the ROC curve, the area under the curve (AUC) obtained was 0.63 (Cl 95\% 0.55 - 0.7), with no cut-off value with a reasonable sensitivity or specificity to be considered. Patients who developed bacteremia had statistically higher CRP levels at $D+6, D+9$, and $D+11$ than patients without bacteremia ( $p<0.05$ for all).

Patients with severe mucositis had higher median CRP levels on $D+6, D+9$ and after $D+11$ ( $p<0.05$ for all). The best linear relationship between CRP and mucositis grade was obtained with the D+6 CRP $(r=0.4 ; p<0.01)$. Median $D+6$ CRP in patients with severe mucositis was higher compared to those with grade 1 and 2 ( 14.9 and $2.9 \mathrm{mg} / \mathrm{dl}$, respectively; $p<0.001$ ), and the AUC obtained was 0.76 (Cl 95\% $0.69-0.83)$.

Length of both neutropenia and hospitalization had statically significant correlations with D+3, D+6, D+ 9 and after D+11 CRPs. The stronger linear relation was obtained with the $D+6 \operatorname{CRP}(r=0.39 ; p<0.001$ for both outcomes).

When comparing the CRP of patients that died with those discharged, there was an associated-on D+9 (19 vs. $7.9 \mathrm{mg} / \mathrm{dL}$ ) and $\mathrm{D}+11$ (14.5 vs. $3.4 \mathrm{mg} / \mathrm{dL}$ ) CRP levels ( $p<0.01$ for both). The D+9 CRP AUC was 0.83 $(95 \% \mathrm{Cl} 0.7-0.95)$ to predict mortality (Figure 2$)$, and a cut-off of $8.5 \mathrm{mg} / \mathrm{dL}$ the D+9 CRP had $83 \%$ and $79 \%$ of sensitivity and specificity, respectively.

The CRP variation from $D+3$ to-D+6 was associated with bacteremia, severe mucositis, and length of neutropenia and hospitalization ( $p$ values $<0.01$ ). Areas obtained by the ROC curve were similar to those reached with single point CRP values, for instance, CRP variation from $d+3$ to $D+6$ and FN CRP had both AUC of 0.62 to predict bacteremia. For severe mucositis, CRP variation from $D+3$ to $D+6$ and $D+6 C R P$ had both AUC 0.7. Although the CRP variation from $D+3$ to D+6 was not statistically associated with mortality, in patients who died and were discharged it was 9.3 vs. $3.2 \mathrm{mg} / \mathrm{dL}(\mathrm{p}=0.62)$, respectively.

\section{DISCUSSION}

Our study was intended to describe the correlation between the CRP absolute values and variations in its dynamic in patients undergoing HSCT, and to search for possible cut off values for prognostic outcomes. We found a correlation with a four-fold increase between the median variation of CRP from $D+3$ and $D+6$ and the outcomes of mucositis (grade 3 and 4), bacteremia, increased neutropenia duration, and more extended hospitalization. We also found a statistically significant correlation between the D+9 CRP and death $(>8.5 \mathrm{mg} / \mathrm{dL}$ with $83 \%$ and $79 \%$ respectively of sensitivity and specificity), but no reasonable cut off value on the ROC curve was noted.

Regarding preconditioning CRP values, we found no correlation with any outcomes we were studying. The literature has some confronting data over the CRP predicting capabilities when measured before HSCT. AKI et al. found, in a cohort of allogeneic transplan- 
tation patients, that prior conditioning CRP values were associated with validating prognostic scores (HCT-Cl, EBMT), and had a significant impact on overall survival ${ }^{6}$. CRP equal to or higher than $10 \mathrm{mg} / \mathrm{L}$ (or $1.0 \mathrm{mg} / \mathrm{dL}$ ) had a significant effect on overall survival, as well as serum ferritin and the $\mathrm{HCT}-\mathrm{Cl}$ risk score. Another study, by Andrew S. Artz et al., confirmed the previous results and suggested levels over than $0.367 \mathrm{mg} / \mathrm{dL}$ as a threshold for transplant-related mortality ${ }^{9}$. In a study performed in lymphoma patients submitted to autologous stem cell transplant, CRP levels before HSCT had significant survival impact with special emphasis on disease status at the procedure ${ }^{10}$. In our data, including only autologous recipients, prior conditioning CRP was over $0.3 \mathrm{mg} /$ $\mathrm{dL}$ (or $3 \mathrm{mg} / \mathrm{L}$ ) in $66 \%$ of patients. A significant association with mortality was only observed considering later collected samples (D+9 and D+11 CRP sample). CRP collected on D+9 had the best performance to predict mortality. Considering $8.5 \mathrm{mg} / \mathrm{dL}$ (or $85 \mathrm{mg} / \mathrm{L}$ ) as a cutoff, CRP on D+9 had sensitivity and specificity of $83 \%$ and $79 \%$ respectively. This threshold is very higher than those described by Aki and Artz in allogeneic patients ${ }^{6,9}$.

Regarding febrile neutropenia and bacteremia, despite a significant difference between CRP values of patients with and without bacteremia, no cut off value had a good performance to predict the outcome. The same results were observed for severe grades of mucositis. The role of systemic inflammatory markers in febrile neutropenia was addressed in several studies ${ }^{11-14}$, with conflicting results. These studies demonstrated that, although CRP levels were higher in patients with complicated febrile neutropenia episodes than non-complicated episodes, there were better markers to be applied, such as procalcitonin (PCT), presepsin, and others. A meta-analysis reported by Wu et cols ${ }^{15}$, concluded that PCT was a highly specific but less sensitive marker of bacterial infection in patients with FN, while CRP was a highly sensitive but less specific marker for bacterial infection. In a study by Karin SR Massaro et al. [14], CRP was compared to procalcitonin (PCT) in febrile neutropenic patients, and PCT levels had a better association with severe infection than CRP concentration to distinguish presence and absence of disseminated infection, but neither biomarkers had an association with mortality. In another study including febrile neutropenia patients, CRP was combined to MASCC risk index to predict the risk of death within 30 days ${ }^{16}$. The combination of the inflammatory parameter (cut-off of $15 \mathrm{mg} / \mathrm{dL}$ ) and the clinical index successfully identified patients with a high risk of death. In a more recent study including only stem cell transplant recipients, Igor Stoma et al. ${ }^{17}$ showed that CRP samples collected 4-hour after the onset of febrile neutropenia were significantly associated with Gram-negative bacteremia. The optimal cut-off value of $16.5 \mathrm{mg} / \mathrm{dL}$ had an average diagnostic value (AUC:0.71) but a low sensitivity (40\%). In this consideration they did not recommend CRP as a routinely biomarker for sepsis. In our study, CRP dynamic variation had interesting associations, with potential clinical applicability. We found a median increment of $3 \mathrm{mg} / \mathrm{dL}$ from the $\mathrm{D}+3$ to the D+6 CRP, and very few patients ( 10\%) had decrease in CRP values during this period. The $D+3-D+6$ variation had a significant correlation with several outcomes (bacteremia, mucositis, duration of neutropenia and hospitalization), but no prediction of mortality. In our data, the CRP variation from D+3 to the CRP from the onset of febrile had no significant association to the development of bacteremia.

The use of antibacterial prophylaxis with quinolones was decided at the discretion of the clinicians. To overcome this limitation, we performed a complementary analysis that revealed no difference in the FN CRP level in patients with or without antibacterial prophylaxis. $(p=0,946)$. Quinolones prophylaxis had no association with occurrence of bacteremia $(p=0,165)$ in this cohort.

This study has some limitations inherent to its retrospective design leading to some missing information from a small number of patients. Nevertheless, the data obtained was considered statistically sufficient to assume relations between the HSCT complications and the dynamic of CRP as pointed out.

The study successfully accessed the dynamic of CRP in HSCT recipients and its association with outcome. CRP levels showed associated with several outcomes, with huge variations. Although there was no cut-off point reasonable to be taken for any of these outcomes, CRP dynamic may be used as possible early red flag markers for patients more prone to complications during HSCT.

\section{CONCLUSION}

CRP levels were associated with bacteremia, mucositis grade, duration of neutropenia and hospitalization, and death. Variation in CRP from $D+3$ to D+6 was an interesting predictor of complications, although the best prediction of mortality was a sample collected on Day+9. 
Compliance with ethical standards

Conflict of interest

The authors declare that they have no conflict of interest.

Ethics approval and consent to participate

This study was approved by institution's Ethical Committees from both centers ("Comitê de Ética em Pesquisa do Hospital Universitário Clementino Fraga Filho" - Reference Number CAAE 51013315.0.0000.5257, and "ProCEP - Comitê de Éti-

\section{REFERENCES}

1. Altshuler C, Haley K, Dhall G, Vasquez L, Gardner SL, Stanek J, Finlay JL. Decreased morbidity and mortality of autologous hematopoietic transplants for children with malignant central nervous system tumors: the "Head Start" trials, 1991-2009. Bone Marrow Transplant. 2016; 51(7):945-8. http://doi.org 10.1038/bmt.2016.45

2. Piñana JL, Montesinos $P$, Martino R, Vazquez $L$, Rovira M, López J, et al. Incidence, risk factors, and outcome of bacteremia following autologous hematopoietic stem cell transplantation in 720 adult patients. Ann Hematol. 2013;93(2):299307. http://doi.org10.1007/s00277-013-1872-4

3. Gil L, Styczynski J, Komarnicki M. Infectious complication in 314 patients after high-dose therapy and autologous hematopoietic stem cell transplantation: risk factors analysis and outcome. Infection. 2007;35(6):421-7. http://doi.org $10.1007 / \mathrm{s} 15010-007-6350-2$

4. Herbers AH, De Haan AF, van der Velden WJ, Donnelly JP, Blijlevens NM. Mucositis not neutropenia determines bacteremia among hematopoietic stem cell transplant recipients. Transpl Infect Dis. 2014;16(2):279-85. http://doi. org10.1111/tid.12195

5. Facchini L, Martino R, Ferrari A, Piñana JL, Valcárcel $D$, Barba $P$, et al. Degree of mucositis and duration of neutropenia are the major risk factors for early post-transplant febrile neutropenia and severe bacterial infections after reduced-intensity conditioning. Eur J Haematol. 2012;88(1):46-51. http://doi.org10.1111/j.16000609.2011.01724.x

6. Akı ŞZ, Suyanı E, Bildacı Y, Çakar MK, Baysal NA, Sucak GT. Prognostic role of pre-transplantation serum C-reactive protein levels in patients with ca em Pesquisa da ESHO Empresa de Serviços Hospitalares - Hospital Pro-Cardiaco"- Reference Number CAAE 51013315.0.3001.5533). All procedures followed were in accordance with the ethical standards of the responsible committee on human experimentation (institutional and national) and with the Helsinki Declaration of 1975, as revised in 2008. The Ethical Committee did not request informed consent as only retrospective data were included and all details that might disclose the identity of the subjects under study should be omitted.

acute leukemia undergoing myeloablative allogeneic stem cell transplantation. Clin Transplant. 2012; 26(5):E513-21. http://doi.org10.1111/ ctr.12028

7. von Lilienfeld-Toal $M$, Dietrich MP, Glasmacher A, Lehmann L, Breig P, Hahn C, et al. Markers of bacteremia in febrile neutropenic patients with hematological malignancies: procalcitonin and IL-6 are more reliable than C-reactive protein. Eur J Clin Microbiol Infect Dis. 2004;23(7):53944. http://doi.org 10.1007/s10096-004-1156-y

8. Prat C, Sancho JM, Domínguez J, Xicoy B, Giménez $M$, Ferrà $C$, et al. Evaluation of procalcitonin, neopterin, C-reactive protein, IL-6 and IL-8 as a diagnostic marker of infection in patients with febrile neutropenia. Leuk Lymphoma. 2008;49(9):1752-61. http://doi.org 10.1080/10428190802258956

9. Artz AS, Logan B, Zhu X, Akpek G, Bufarull RM, Gupta V, et al. The prognostic value of serum C-reactive protein, ferritin, and albumin prior to allogeneic transplantation for acute myeloid leukemia and myelodysplastic syndromes. Haematologica. 2016;101(11):1426-33. http://doi. org10.3324/haematol.2016.145847

10. Ariizumi H, Saito B, Uto Y, Hattori N, Nakashima $H$, Yanagisawa $K$, et al. Predictive role of levels of soluble interleukin-2 receptor and C-reactive protein in selecting autologous PBSC transplantation for lymphoma. Bone Marrow Transpl. 2015;50(2):301-3. http://doi.org 10.1038/ bmt.2014.236

11. Persson $L$, Engervall $P$, Magnuson $A$, Vikerfors $T$, Söderquist B, Hansson L-O,et al. Use of inflammatory markers for early detection of bacteraemia in patients with febrile neutropenia. Scand J Infect Dis. 2004; 
12. Persson L, Söderquist $B$, Engervall $P$, Vikerfors T, Hansson LO, Tidefelt U. Assessment of systemic inflammation markers to differentiate a stable from a deteriorating clinical course in patients with febrile neutropenia. Eur J Haematol. 2005;74(4):297-303. http://doi.org 10.1111/j.1600-0609.2004.00387.x

13. Uys A, Rapoport BL, Fickl H, Meyer PW. Prediction of outcome in cancer patients with febrile neutropenia: comparison of the Multinational Association of Supportive Care in Cancer risk-index score with procalcitonin, C-reactive protein, serum amyloid A, and interleukins- 1 beta, $-6,-8$ and -10 . Eur J Cancer Care (Engl). 2007;16(6):475-83. http://doi.org 10.1111/j.1365-2354.2007.00780.x

14. Massaro KS, Costa SF, Leone C, Chamone DA. Procalcitonin (PCT) and C-reactive protein (CRP) as severe systemic infection markers in febrile neutropenic adults. BMC Infect Dis. 2007; 7(1):137. http://doi.org/10.1186/1471-2334-7-137
15. Wu CW, Wu JY, Chen CK, Huang SL, Hsu SC, Lee $M T$, et al. Does procalcitonin, C-reactive protein, or interleukin- 6 test have a role in the diagnosis of severe infection in patients with febrile neutropenia? A systematic review and meta-analysis. Support Care Cancer. 2015;23(10):2863-72. http://doi.org10.1007/s00520-015-2650-8

16. Combariza JF, Lombana M, Pino LE, Arango M. C-reactive protein and the MASCC risk index identify high-risk patients with febrile neutropenia and hematologic neoplasms. Support Care Cancer. 2015;23(4):1009-13. http://doi.org $10.1007 / \mathrm{s} 00520-014-2454-2$

17. Stoma I, Karpov I, Uss A, Rummo O, Milanovich $\mathrm{N}$, Iskrov I. Diagnostic value of sepsis biomarkers in hematopoietic stem cell transplant recipients in a condition of high prevalence of gram-negative pathogens. Hematol Oncol Stem Cell Ther. 2017;10(1):15-21. http://doi.org 10.1016/j. hemonc.2016.09.002

TABLE 1: Characteristics of 340 autologous stem cell transplants

\begin{tabular}{|c|c|}
\hline & $\mathrm{N}=340$ \\
\hline \multicolumn{2}{|l|}{ CENTER, $\mathrm{N}(\%)$} \\
\hline 1 & $51(15)$ \\
\hline 2 & $289(86)$ \\
\hline \multicolumn{2}{|l|}{ CONDITIONING REGIMEN, N (\%) } \\
\hline MELPHALAN & $211(62)$ \\
\hline BEAM & $111(33)$ \\
\hline BUCYVP & $10(3)$ \\
\hline CBV & $4(1)$ \\
\hline OTHERS & $4(1)$ \\
\hline FEBRILE NEUTROPENIA, N (\%) & $299(88)$ \\
\hline UNKNOWN ORIGIN & $192(64)$ \\
\hline CLINICALLY DOCUMENTED & $24(8)$ \\
\hline MICROBIOLOGICALLY DOCUMENTED & $83(28)$ \\
\hline WITHOUT BACTEREMIA & 3 \\
\hline WITH BACTEREMIA* & 80 \\
\hline DUE TO GRAM NEGATIVE BACTERIA & 35 \\
\hline DUE TO GRAM POSITIVE BACTERIA & 46 \\
\hline \multicolumn{2}{|l|}{ MUCOSITIS, N=236 (\%) } \\
\hline MUCOSITIS > GRADE 2 & $171(72)$ \\
\hline MUCOSITIS > GRADE 3 & $61(26)$ \\
\hline DURATION OF NEUTROPENIA IN DAYS, MEDIAN (RANGE) & $6(3-36)$ \\
\hline DURATION OF HOSPITALIZATION IN DAYS, MEDIAN (RANGE) & $19(8-64)$ \\
\hline DEATH, $N(\%)$ & $7(2)$ \\
\hline
\end{tabular}

BEAM: BCNU, etoposide, cytarabine and melphalan; CBV: cyclophosphamide, carmustine and etoposide; BuCyVP: cyclophosphamide, etoposide and busulfan *One patient had both Gram-negative and Gram-positive bacteremia 
TABLE 2: CRP levels according to sample collection time related to transplant

\begin{tabular}{c|c}
\hline Time related to infusion (Dzero) & Median (range), $\mathrm{mg} / \mathrm{dL}$ \\
\hline Before $\mathrm{HSCT}, \mathrm{n}=238$ & $0.48(<0.01-15)$ \\
\hline $\mathrm{D}$ Zero, $\mathrm{n}=239$ & $0.38(<0.01-20)$ \\
\hline $\mathrm{D}+3, \mathrm{n}=274$ & $0.71(<0.01-28)$ \\
\hline $\mathrm{D}+6, \mathrm{n}=321$ & $4.66(<0.01-32,2)$ \\
\hline $\mathrm{D}+9, \mathrm{n}=318$ & $8.07(<0.01-32.3)$ \\
\hline After $\mathrm{D}+11, \mathrm{n}=276$ & $3.49(<0.01-31.5)$ \\
\hline Onset of $\mathrm{FN}, \mathrm{n}=298$ & $3.30(<0.01-27.2)$ \\
\hline Engraftment, $\mathrm{n}=321$ & $4.93(<0.01-31.2)$ \\
\hline
\end{tabular}

FN: febrile neutropenia; CRP: C-reactive protein

FIGURE 1: CRP variation during autologous stem cell transplant

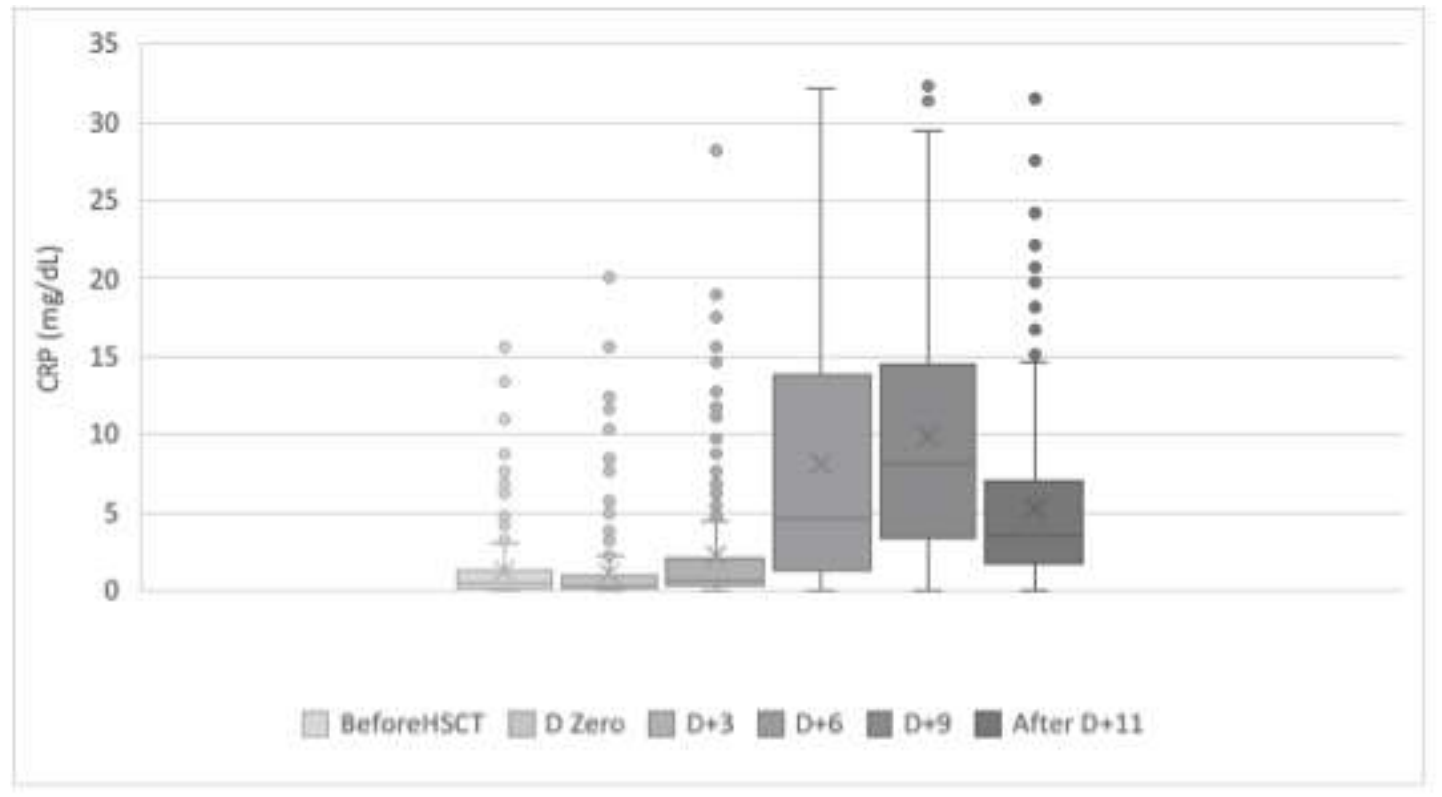

CRP: C-reactive protein; HSCT: hematopoietic stem cell transplant

FIGURE 2: ROC Curve showing the performance of CRP D+9 levels to predict mortality

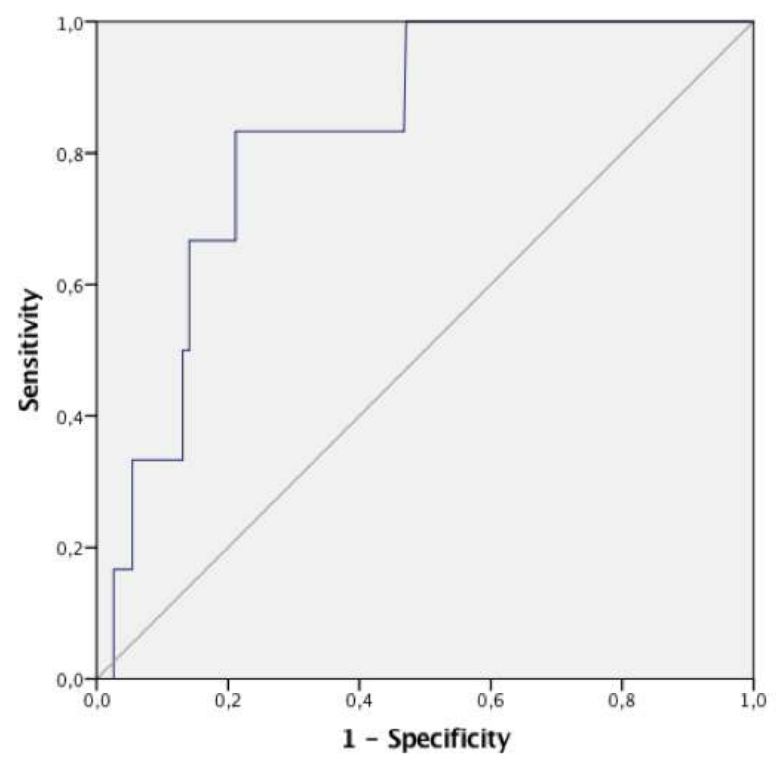

